\title{
OPTIMIZATION OF MULTI-TASK JOB-SHOP SCHEDULING BASED ON UNCERTAINTY THEORY ALGORITHM
}

\author{
Chen, Q.*; Deng, L. F.**\# \& Wang, H. M. \\ * The School of Economics and Trade, Hunan University, Changsha 410082, China \\ ** Centre for Economics, Finance and Management Studies, Hunan University, Changsha 410082, China \\ ${ }^{* * * *}$ Shaoxing University, Shaoxing 312000, China \\ E-Mail: paperway@sina.cn, denglanfang1987227@126.com,glanme@126.com \\ ( ${ }^{\#}$ Corresponding author)
}

\begin{abstract}
This paper aims to solve the difficulties in job-shop scheduling, a key determinant of production cost and economic effectiveness of manufacturing enterprises. For this purpose, the existing job-shop scheduling problems were introduced, the internal and external uncertainties that may influence the scheduling plan in actual production were enumerated, and the corresponding scheduling and rescheduling strategies were reviewed one by one. Then, the flexible job-shop model was established under uncertainties, the mathematical models were created considering such three uncertainties as the discrete makespan, rush order cut-in and machine failure, and the proactive-reactive scheduling strategy was proposed against emergencies or uncertainties. Finally, the proactive-reactive scheduling strategy, developed based on the uncertainty theory, was proved effective and feasible through the simulation experiments on scheduling optimization under machine failure and rush order cut-in. The research findings shed new light on the production planning and scheduling strategy of manufacturing enterprises.

(Received, processed and accepted by the Chinese Representative Office.)
\end{abstract}

Key Words: Uncertainty Theory, Multi-Task Job-Shop Scheduling, Scheduling Optimization, Economic Effectiveness

\section{INTRODUCTION}

Economic globalization and technological development are a mixed blessing for manufacturing, the foundation of national economy [1]. The manufacturing industry is faced with both rare opportunities and enormous challenges. With the continuous improvement of living standards, the customers have been raising increasingly diverse, subjective and individualized demands for products [2], while the emergence of new techniques have greatly accelerated the renewal of products. Overall, the traditional mass production is shifting towards modern production modes like lean production [3]. The modern production modes are featured by automated process, diverse products and small and medium batches. These new phenomena have added to the difficulty in job-shop scheduling $[4,5]$. In addition to advanced production technology, the enterprises must rely on cutting-edge production plan and production control to adapt to the modern production modes in a rapid and stable manner and survive the intense competition in the market $[6,7]$.

Job-shop scheduling, as the core production technology, directly bears on the production cost and efficiency of the enterprises, and determines whether a modern enterprise could run efficiently and stably and maintain market competitiveness [8]. Through rational job-shop scheduling, it is possible to achieve the following goals under multiple constraints and limited resources [9]: the minimal processing cost, the maximum profit, the highest consumer satisfaction and the best utilization rate of the machines. The previous studies have shown that, the actual processing time only takes up $8 \%$ of the entire cycle of product manufacturing, while the remaining parts belong to waiting for processing, transport and storage [10]. 
Therefore, it is very meaningful for enterprises to optimize their job-shop scheduling [11]. In actual practice, however, there are many uncertainties and emergencies to be tackled in jobshop scheduling $[12,13]$. If not solved timely, these factors may endanger the production efficiency and smoothness.

To solve the difficulties in job-shop scheduling, this paper probes deep into the relevant optimization methods based on the uncertainty theory and proposes a systematic solution for enterprises to optimize the internal and external resources, maximize their economic effectiveness, coordinate the multiple production tasks in the job-shop, and overcome uncertainties and emergencies [14].

\section{OVERVIEW OF JOB-SHOP SCHEDULING PROBLEM}

\subsection{Job-shop scheduling problem}

Scheduling is a ubiquitous problem. Its presence is seen in such fields as transport, manufacturing, hospital care, etc. [15]. The goal of scheduling lies in completing a job or realizing a goal within a limited time using the minimum amount of resources. As an important scheduling problem, the job-shop scheduling problem refers to the completion of the production task according to the required time, quantity and quality through the rational arrangement of the processing time and resources for a separable task under certain constraints like makespan and sequence [16]. The purpose is to improve the productivity, shorten the production cycle, reduce the production cost and maximize the profits of the enterprise. The common constraints, i.e. goals, of job-shop scheduling are described in Fig. 1.

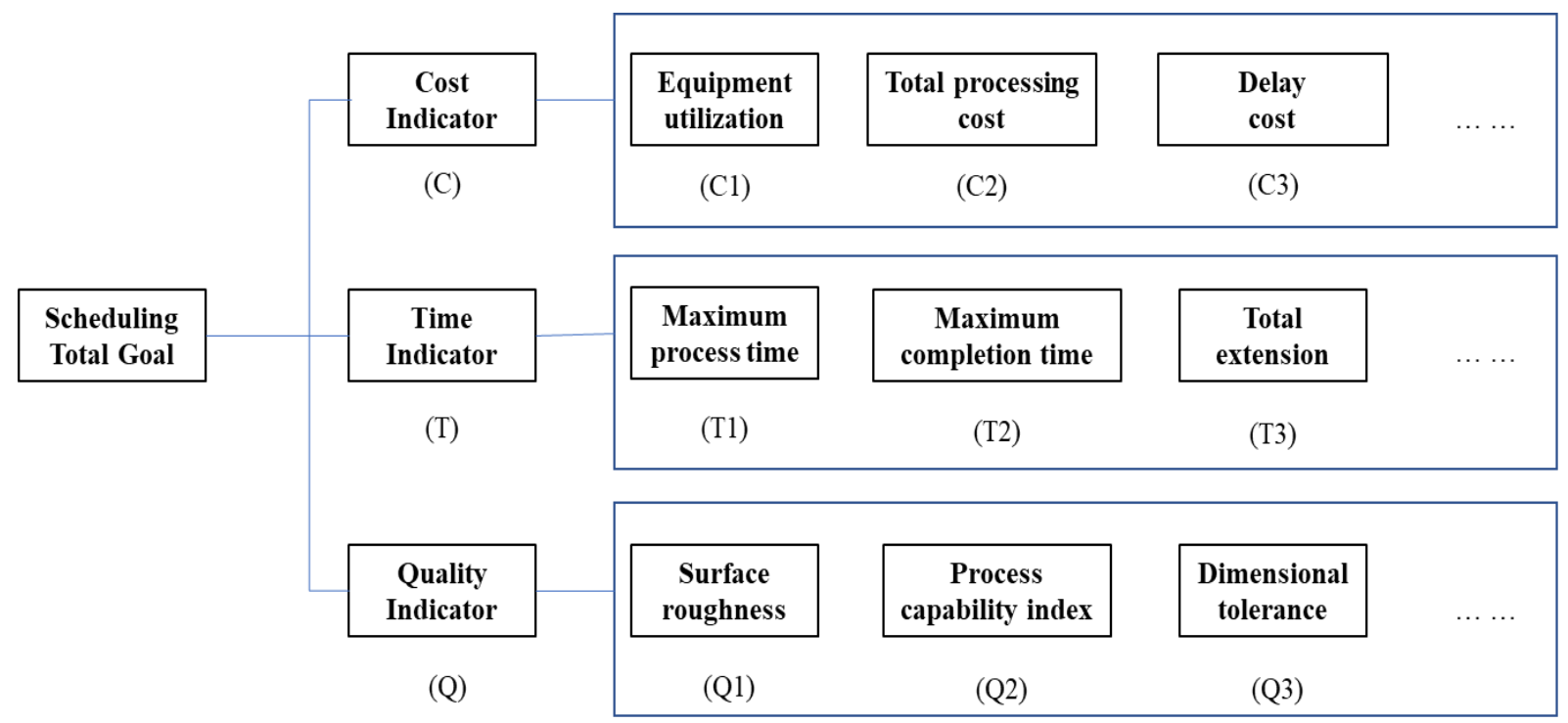

Figure 1: Common constraints of job-shop scheduling.

\subsection{Features of job-shop scheduling}

The various classification methods for job-shop scheduling problems are based on the number of resource constraints, job-shop composition, or processing features. Whichever the basis, all job-shop scheduling problems share similar basic features (Fig. 2). Featured by largescale production, multiple processes and numerous departments, the multi-task job-shop scheduling is a dynamic and complex system involving multiple levels and multiple factors. 


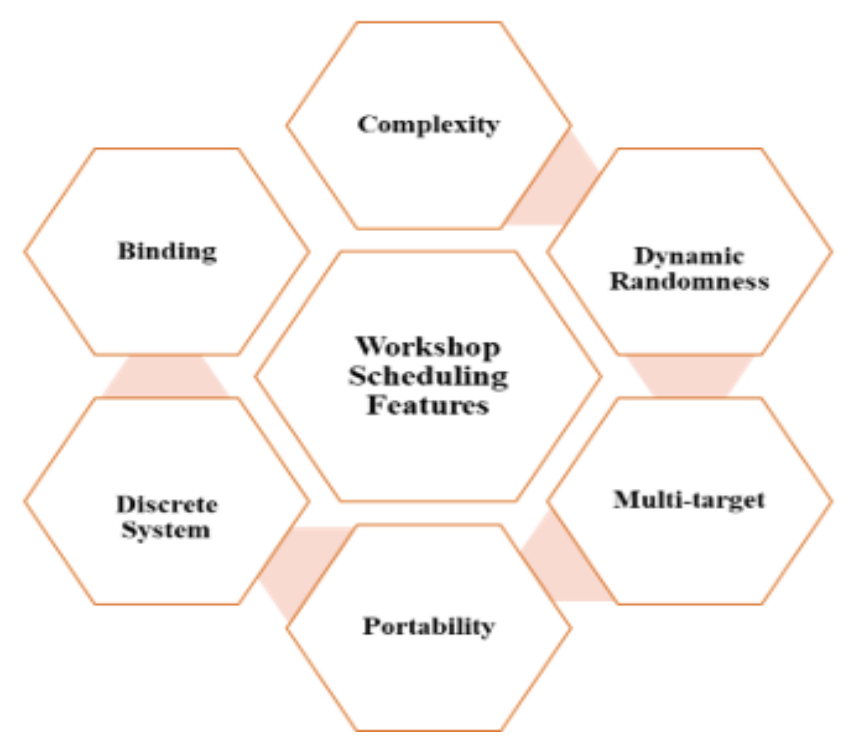

Figure 2: Basic features of job-shop scheduling problems

\section{UNCERTAINTY THEORY}

\subsection{Scheduling decision mechanism}

Manufacturing is a dynamic process influenced by various uncertainties. Under uncertain factors, the scheduling problem is no longer the search for the optimal solution, but a methodological issue involving the preferences and attitudes of different decision makers. In light of the interferences of the job-shop, the uncertainties can be divided into internal factors and external factors (Fig. 3).

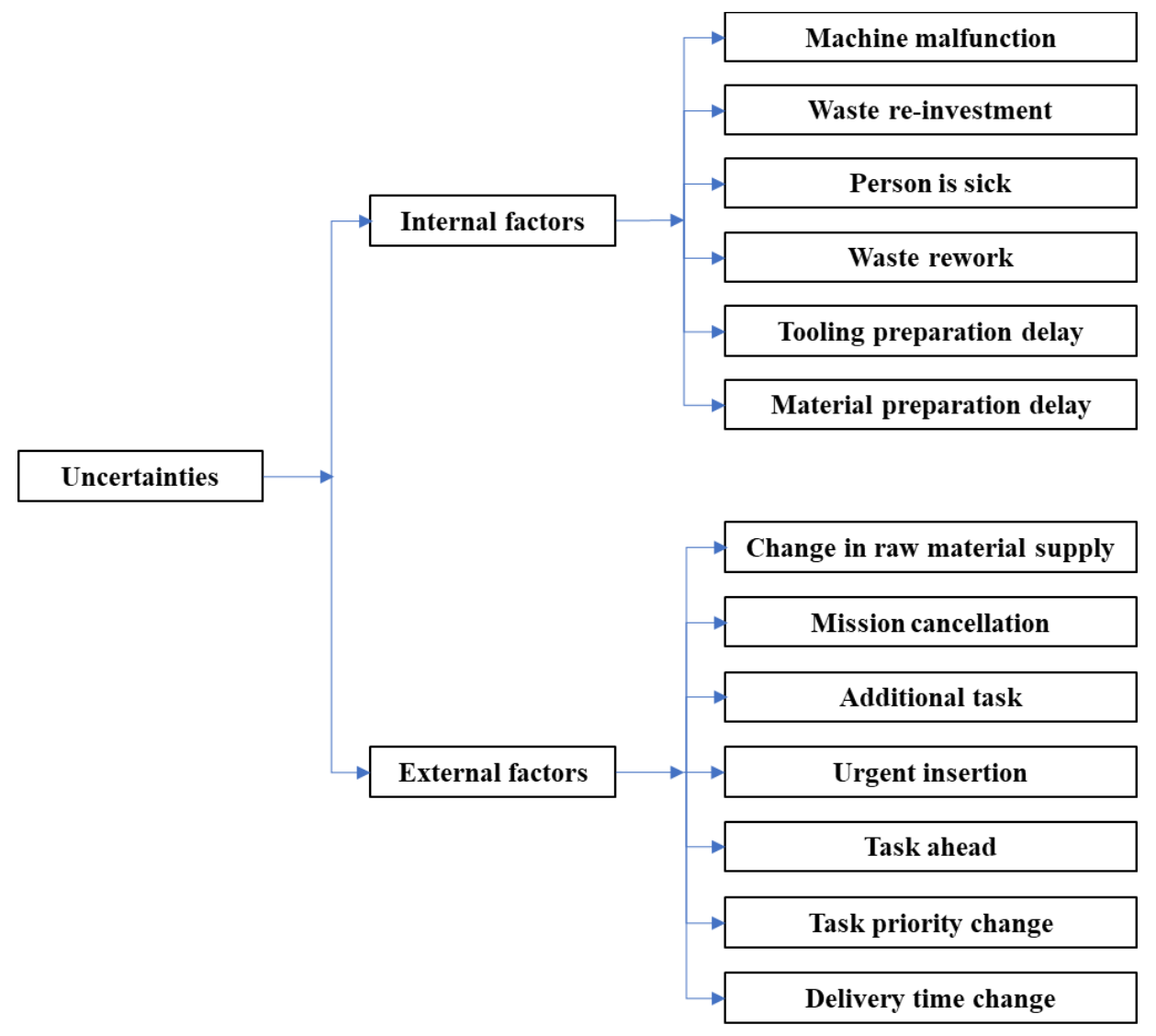

Figure 3: Classification of job-shop uncertainties. 
Based on the said uncertainties, the job-shop scheduling strategies can be categorized into proactive scheduling, reactive scheduling, fuzzy scheduling and hybrid scheduling. Specifically, the proactive scheduling considers the uncertainties in the preparation of the scheduling plan; the reaction scheduling makes timely adjustment to the scheduling plan according to the emergencies or uncertainties; the fuzzy scheduling is relatively flexible due to its fuzzy features under uncertainties; the hybrid scheduling solves the problems caused by uncertainties through the combination of the former three strategies.

\subsection{Scheduling indices}

As shown in Fig. 4 below, scheduling is mainly measured by four indices, including the flexibility, stability, executability and robustness. During the manufacturing, flexibility reflects how flexible the time, processing mode or sequence in the execution of the scheduling plan. The stability of the scheduling plan has a major impact on the plan execution, material demand and external coordination in the production process. Besides ensuring flexibility, the adjusted scheduling plan should not deviate far from the original plan. Robustness means the effect of the scheduling plan is immune to any interference or uncertainty. Executability requires that no constraint is violated by the execution of the scheduling plan. It is difficult to maintain the executability in actual practice, owing to the presence of emergencies and uncertainties.

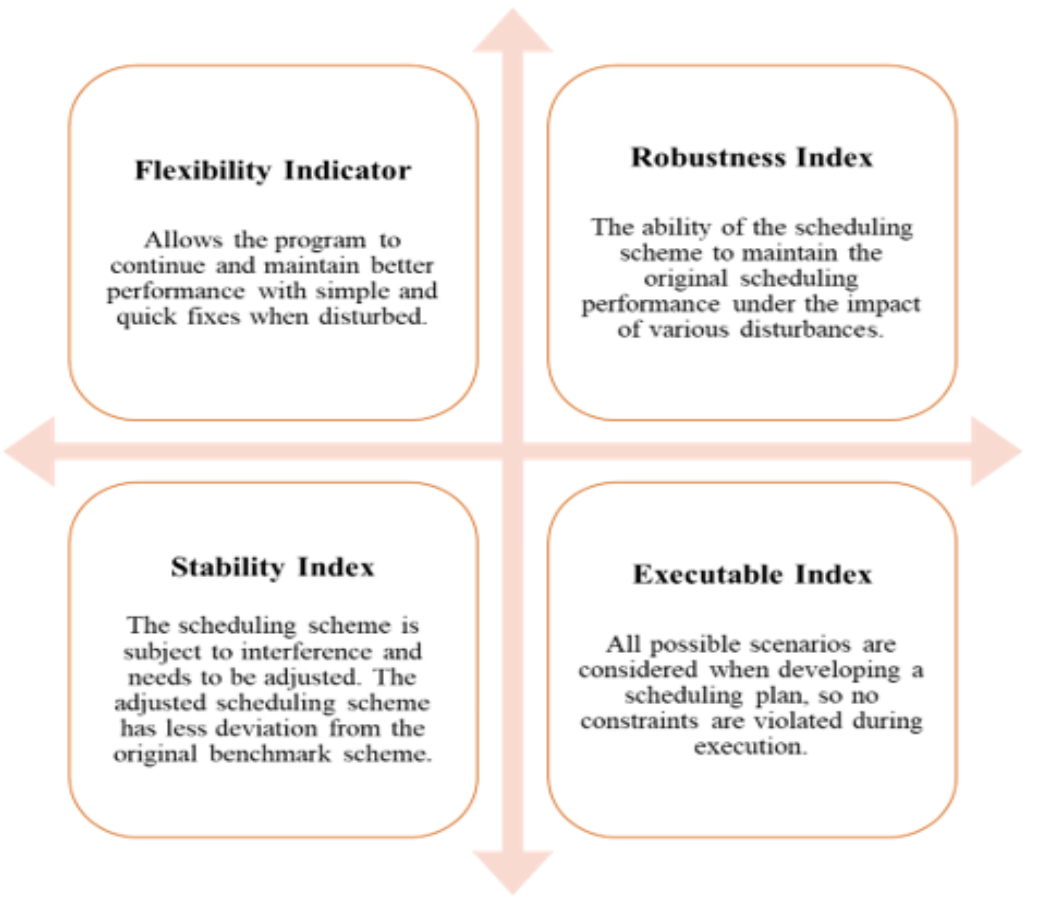

Figure 4: Scheduling indices.

\subsection{Rescheduling strategy under uncertainties}

The original scheduling plan may not be implemented smoothly due to the numerous uncertainties in each link of actual job-shop production, especially in multi-task production mode. Thus, a single scheduling strategy is not flexible enough to meet the requirements for actual production. Currently, many scholars have combined reactive and proactive scheduling strategies to solve actual scheduling problems, but failed to determine the proper timing of scheduling. As is known to all, excessively frequent rescheduling will harm the scheduling stability, while untimely rescheduling cannot repair the system. In general, the rescheduling strategies can be roughly classified into three types (Fig. 5). 


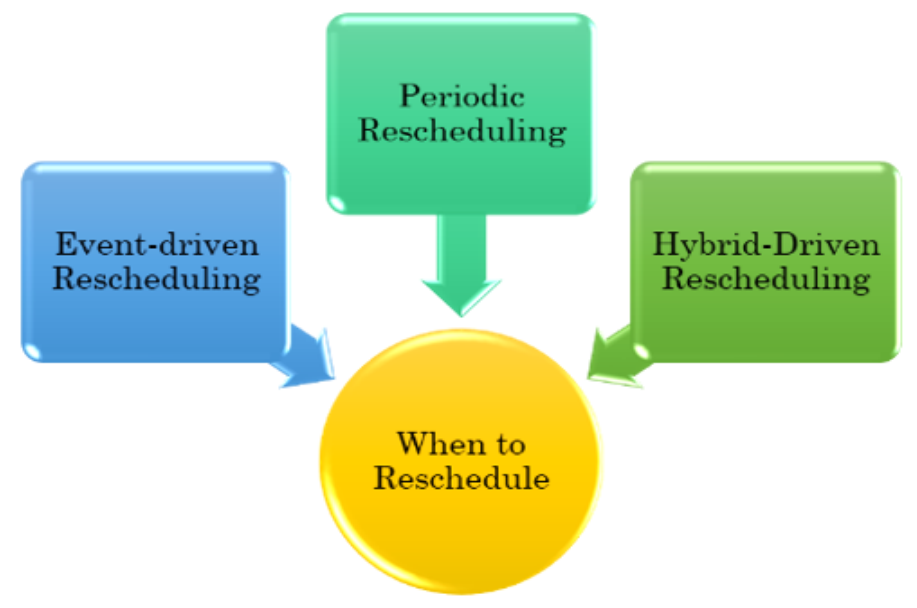

Figure 5: Rescheduling strategies.

\section{OPTIMIZATION OF MULTI-TASK JOB-SHOP SCHEDULING BASED ON UNCERTAINTY THEORY ALGORITHM}

\subsection{Flexible job-shop modelling under uncertainties}

The diversification of consumer demands has given birth to small-batch, multi-variety flexible manufacture system. During workpiece manufacturing, the variety of processing paths, sequences and machines helps enhance the production stability, improve the utilization rate of machines and shorten the makespan. Hence, the multi-task, multi-objective flexible job-shop scheduling has great application significance. As a result, the uncertainties of flexible jobshop were modelled in the uncertain environment.

(1) Discrete probability model for makespan

Due to the makespan uncertainty of each workpiece, the makespan can be regarded as some discrete points between the maximum and the minimum makespans. Thus, the discrete probability model for makespan can be established as:

$$
\varphi(\mathrm{A})= \begin{cases}P_{1}(\mathrm{~A}), & \mathrm{c}=c_{1}(A) ; \\ P_{2}(\mathrm{~A}), & \mathrm{c}=c_{2}(A) \\ & \cdots \\ P_{Q}(\mathrm{~A}), & \mathrm{c}=c_{Q}(A)\end{cases}
$$

where $c_{1}(A)$ is the makespan for workpiece $\mathrm{A} ; P_{1}(\mathrm{~A})$ is the probability of the makespan. Note that $\sum_{i=1}^{Q} P_{1}(\mathrm{~A})=1$ and $c_{1}(A)<c_{2}(A)<\ldots<c_{Q}(A)$.

(2) Failure models

Two mathematical models were created based on the failure rate and repair rate of the machines. It is assumed that there are $a^{k}$ machines belonging to type $k$, and $j^{k}$ machines that have been shut down. Let $f=1 /$ MTTF be the rate of single machine failure and $r=1 / M T T R$ be the repair rate of single machine failure. Note that the $M T T F$ is short for the mean time to failure, and the MTTR is short for the mean time to repair. Then, the total failure rate and repair rate of $a^{k}-j^{k}$ machines can be expressed as:

$$
\begin{aligned}
& f_{j}=\cup\left(a^{k}-j^{k}\right) f \\
& r_{j}=\cup\left(a^{k}-j^{k}\right) r
\end{aligned}
$$

(3) Rush order cut-in model

Assuming that the rush order cut-in obeys the uniform distribution, the following model can be established: 


$$
f(x)=\left\{\begin{array}{cl}
\frac{1}{b-a}, & a \leq x \leq b \\
0, & \text { others }
\end{array}\right.
$$

where $a$ and $b$ are two types of rush orders.

\subsection{Proactive-reactive scheduling process based on uncertainty theory}

Based on the uncertainty theory, the proactive and reactive scheduling strategies were combined to solve multi-task, multi-objective flexible job-shop scheduling problems. The flow of the integrated strategy consists of three parts (Fig. 6):

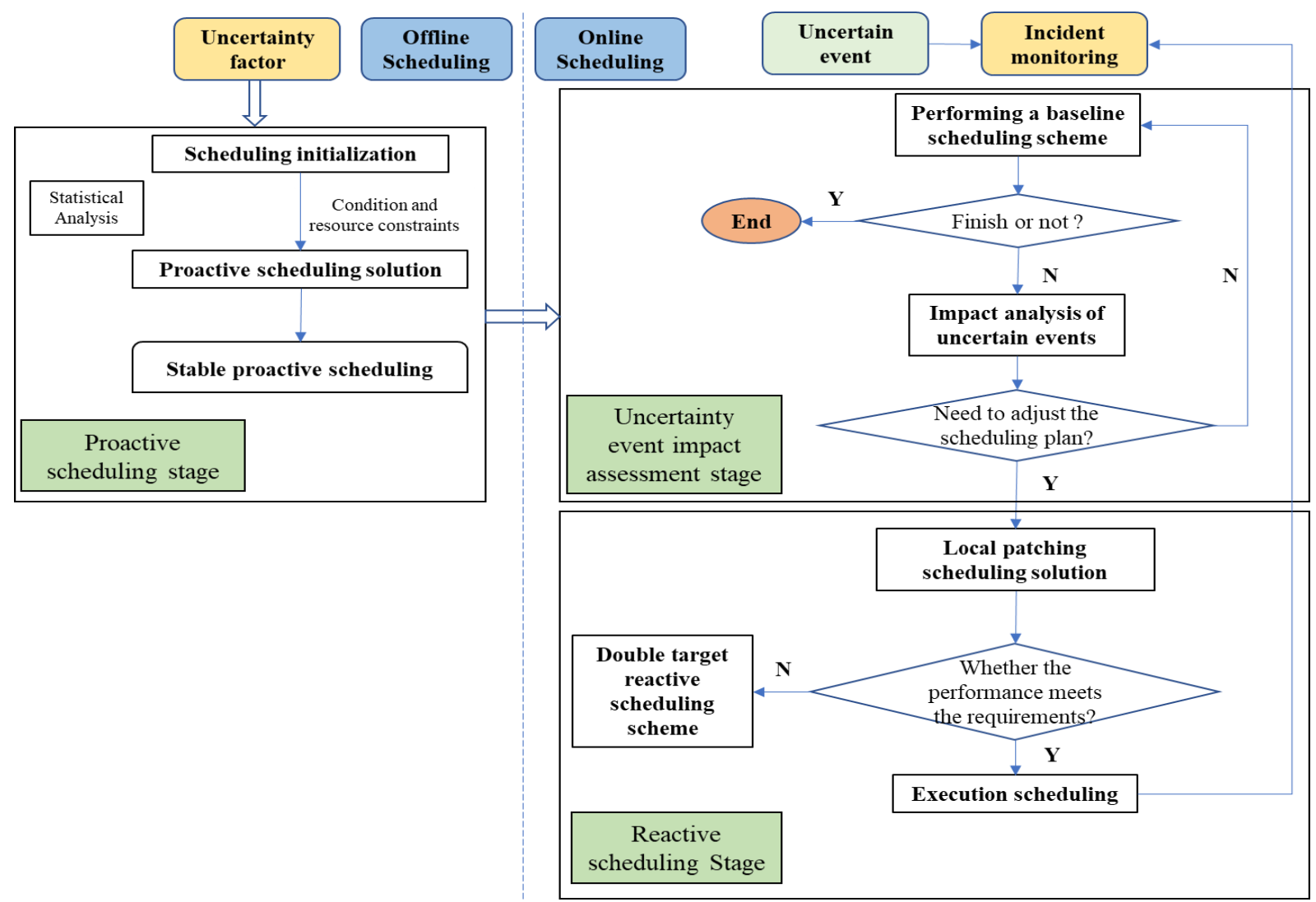

Figure 6: The three phases of the integrated scheduling strategy.

(1) The proactive scheduling phase: During the design of the scheduling plan, a stable proactive plan was constructed based on the discrete probability model for makespan, considering the uncertain makespan factors, and was adopted as the benchmark plan for scheduling.

(2) The evaluation of rescheduling time points: Despite a certain anti-interference ability, the original scheduling plan may be affected by emergencies in actual production. Thus, it is necessary to determine when to start the rescheduling strategy.

(3) The reactive scheduling phase: The scheduling plan was modified in local parts to cope with the impacts from emergencies or uncertainties. If the impacts were not fully dampened, the reactive scheduling was implemented in a comprehensive manner.

\subsection{Experimental simulation and results analysis}

The proposed integrated scheduling strategy was validated through Matlab simulation experiment on multi-task job-shop scheduling. The scheduling aims to achieve the minimal energy consumption and the shortest makespan. During the experiment, it is assumed that 12 
workpieces need to be processed in a job-shop through 3 processes on 9 machines. Two uncertainties were taken into consideration: (1) three new workpieces were added at $t=9$; (2) a machine failed at $t=10$ and was repaired at $t=11$. Under the premise of meeting the delivery date, the scheduling plan with the least energy consumption was selected. The Gantt chart of the original plan is shown in Fig. 7 below. It can be seen that the makespan was $38 \mathrm{~h}$ and the energy consumption was $264.5 \mathrm{kWh}$ for the minimal energy consumption.

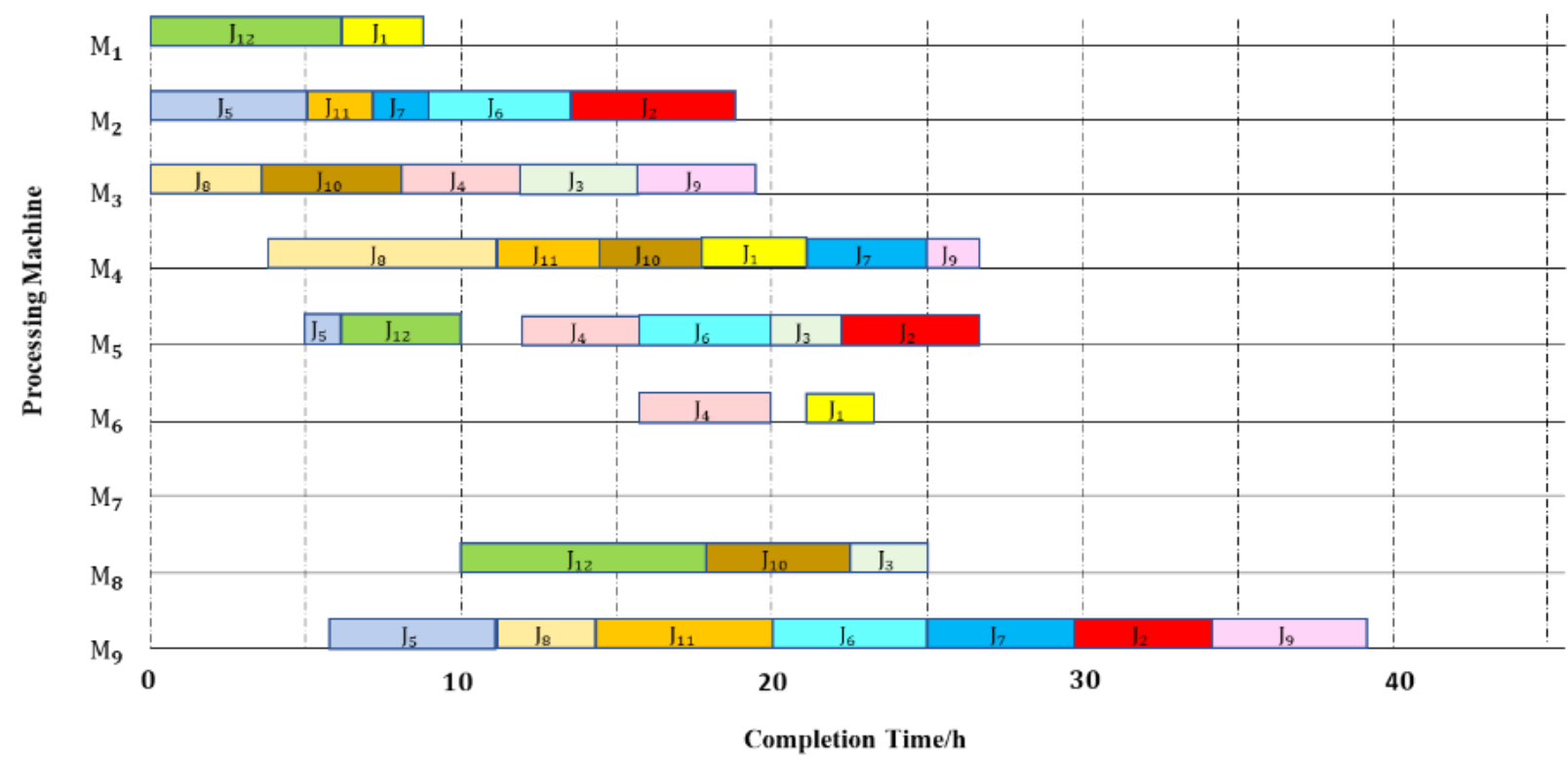

Figure 7: Gantt chart of the original plan.

(1) Rush order cut-in

Two strategies are available for rush order cut-in to the original plan: first, add the new workpieces after each operating machine completes the processing of the workpieces in the corresponding phase, while each nonoperating machine directly processes the new rush orders; second, scheduling the original workpieces and the rush orders simultaneously. The Gantt charts of the two strategies are given in Figs. 8 and 9, respectively. It can be seen that the makespan and energy consumption of the first strategy were $44 \mathrm{~h}$ and $298.6 \mathrm{kWh}$, while those of the second strategy were $46 \mathrm{~h}$ and $280.4 \mathrm{kWh}$.

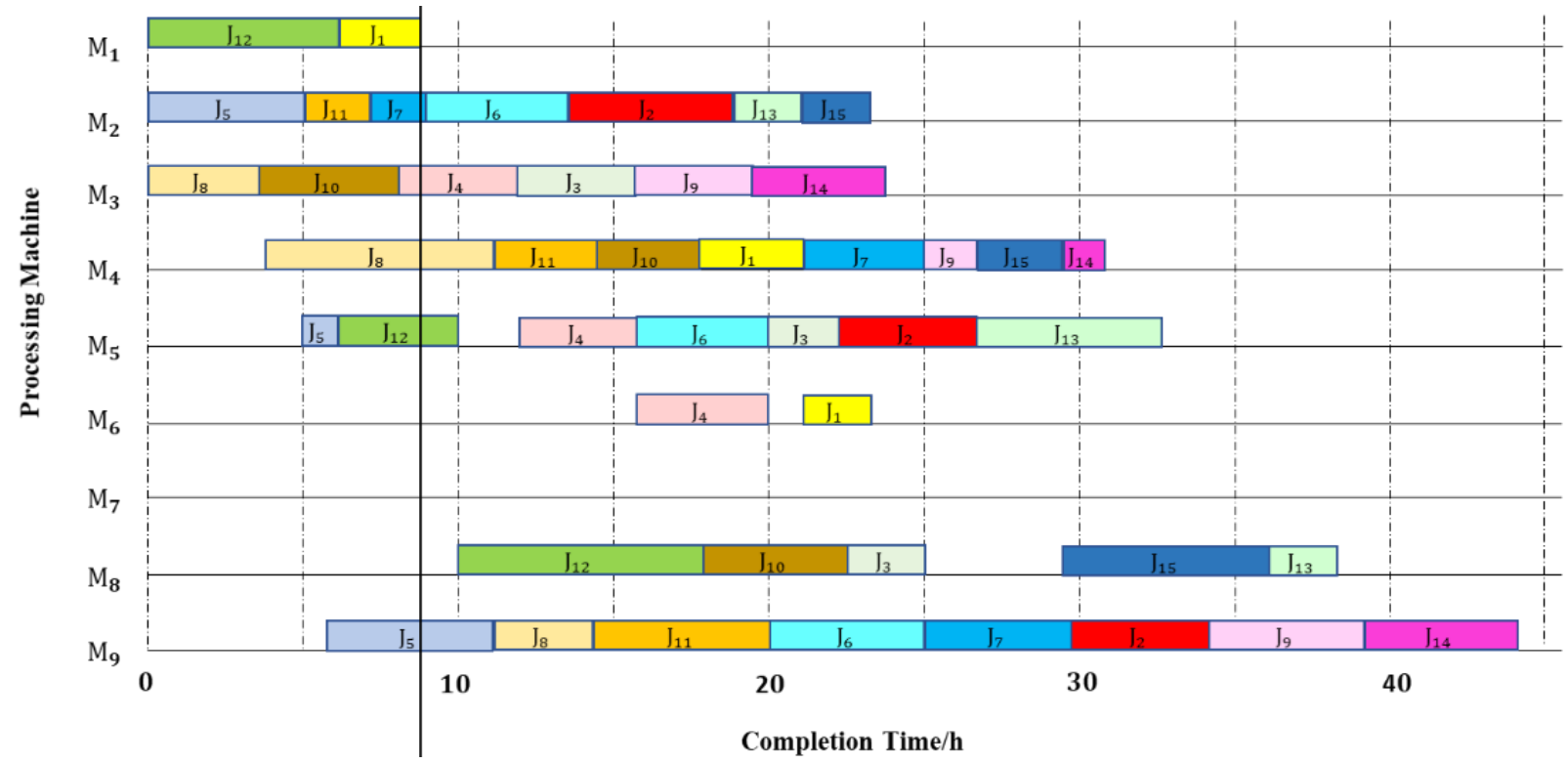

Figure 8: Gantt chart of the original plan after local repair. 


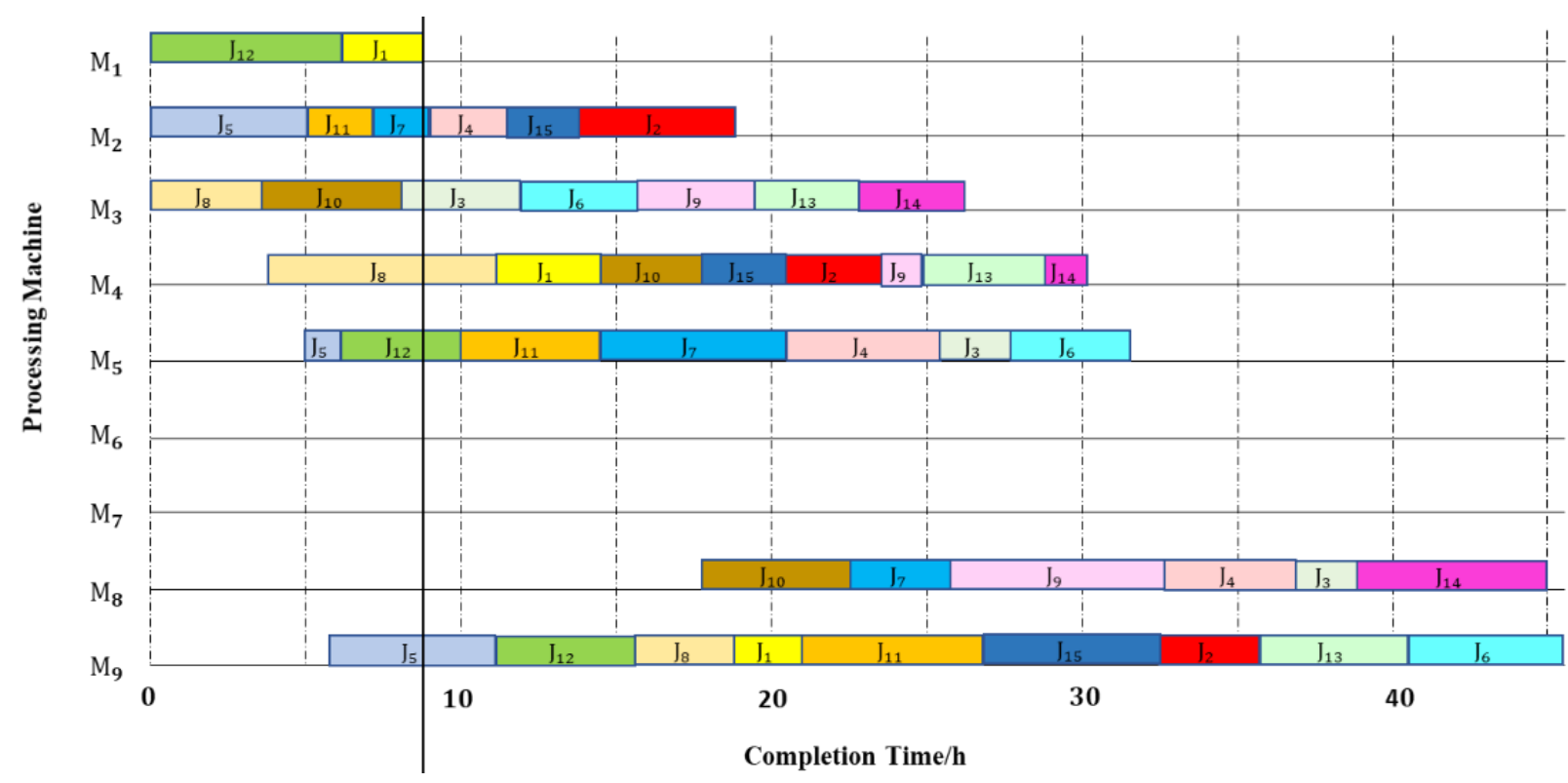

Figure 9: Gantt chart of the original plan after rescheduling.

It is clear that both strategies underwent an increase in energy consumption and makespan. However, Strategy 2 consumed less energy than Strategy 1 under the premise of meeting the delivery date. The comparison between the two strategies is illustrated in Fig. 10.

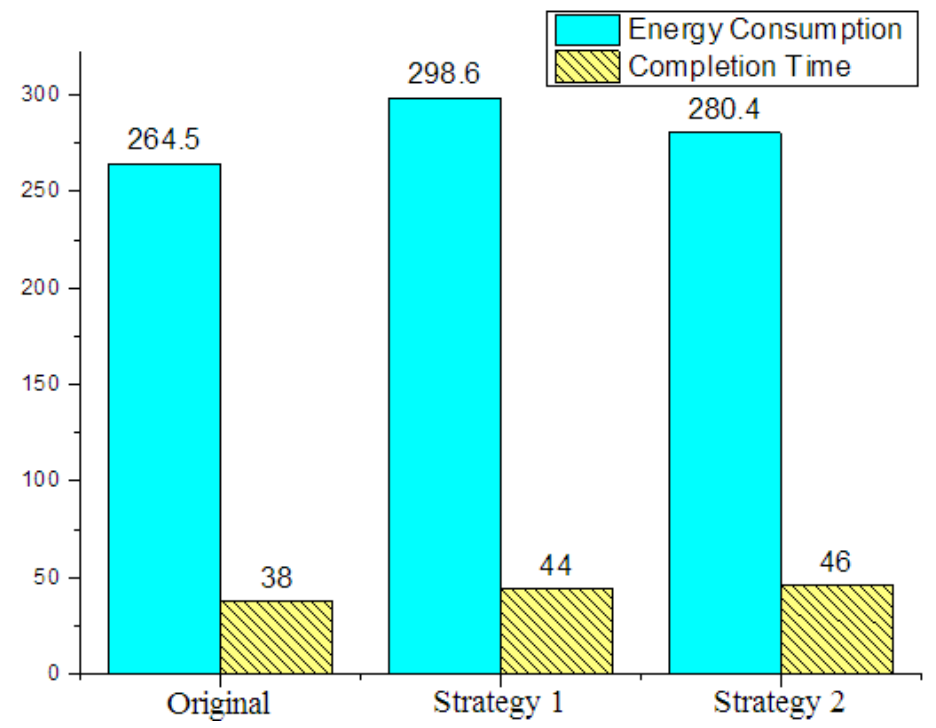

Figure 10: Comparison between the two strategies.

(2) Machine failure

When a machine failed, the workpieces to be processed on that machine were distributed to other machines, especially the nonoperating ones. Based on the uncertainty theory algorithm, the makespan and energy consumption corresponding to the minimal energy consumption were determined as $42 \mathrm{~h}$ and $288.5 \mathrm{kWh}$. The Gantt chart of the corresponding optimal scheduling is shown in Fig. 11. 


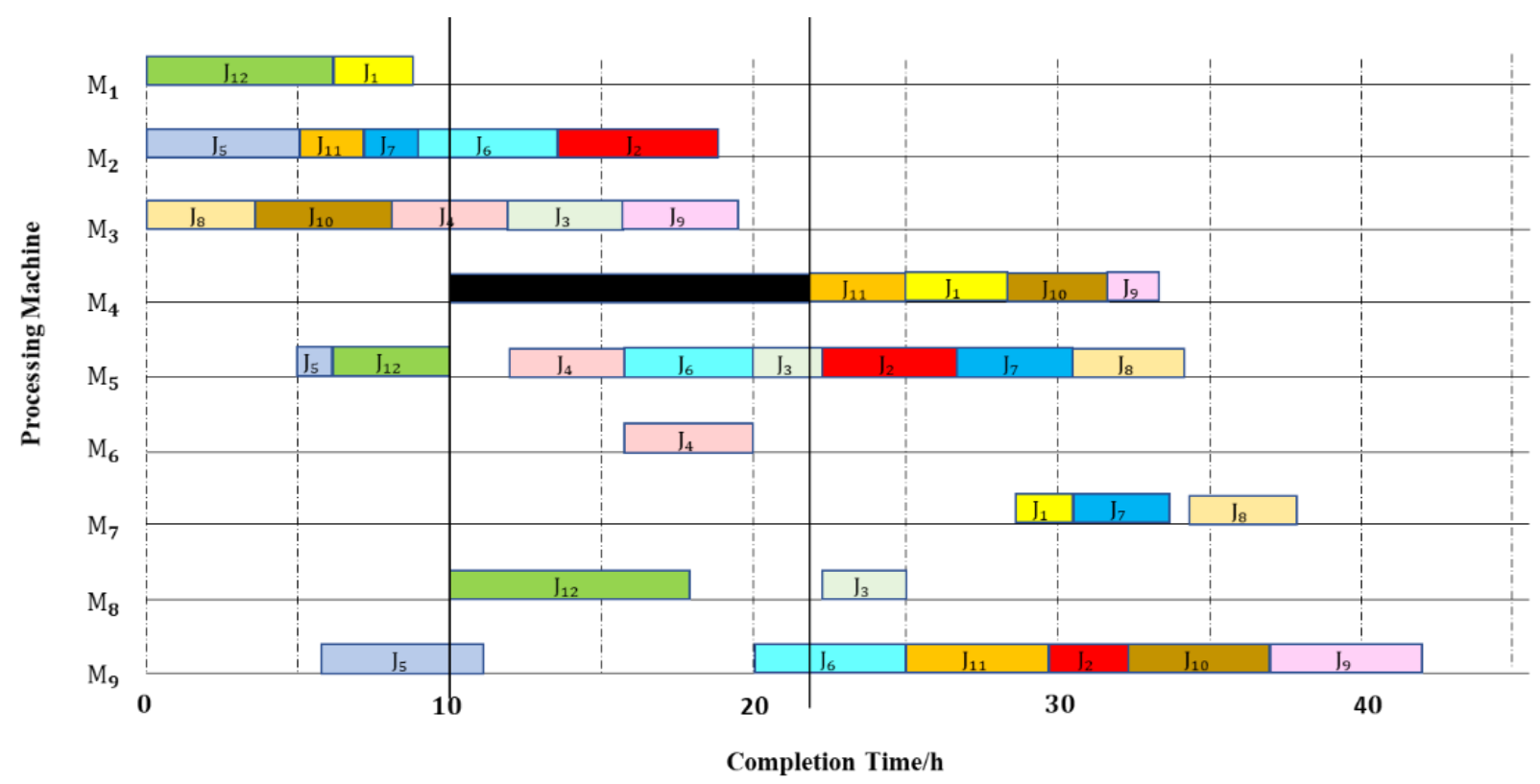

Figure 11: Gantt chart of the original plan under machine failure.

\section{CONCLUSIONS}

Targeting the difficulties in job-shop scheduling, this paper carries out in-depth exploration into the relevant optimization methods based on the uncertainty theory algorithm. The following conclusions were drawn from our research:

(1) The existing job-shop scheduling problems were introduced, the internal and external uncertainties that may influence the scheduling plan in actual production were enumerated, and the corresponding scheduling and rescheduling strategies were reviewed one by one.

(2) The flexible job-shop model was established under uncertainties, the mathematical models were created considering such three uncertainties as the discrete makespan, rush order cut-in and machine failure, and the proactive-reactive scheduling strategy was proposed against emergencies or uncertainties.

(3) The proactive-reactive scheduling strategy, developed based on the uncertainty theory, was proved effective and feasible through the simulation experiments on scheduling optimization under machine failure and rush order cut-in.

\section{ACKNOWLEDGEMENT}

This research was supported by Zhejiang Provincial Natural Science Foundation of China under Grant No. Q14A010009 and Natural Science Foundation of China under Grant No: 11501375.

\section{REFERENCES}

[1] Onuh, S.; Popov, I.; Bennett, N. (2007). Trends in agility for rapid product development and manufacturing - a review, International Journal of Agile Systems and Management, Vol. 2, No. 2, 135-146, doi:10.1504/IJASM.2007.015784

[2] Droste, M.; Deuse, J. (2012). A planning approach for in-plant milk run processes to optimize material provision in assembly systems, ElMaraghy, H. A. (Ed.), Enabling Manufacturing Competitiveness and Economic Sustainability, Springer, Berlin, 604-610, doi:10.1007/978-3-64223860-4_99

[3] Graves, S. C. (1981). A review of production scheduling, Operations Research, Vol. 29, No. 4, 646-675, doi:10.1287/opre.29.4.646 
[4] Adams, J.; Balas, E.; Zawack, D. (1988). The shifting bottleneck procedure for job shop scheduling, Management Science, Vol. 34, No. 3, 391-401, doi:10.1287/mnsc.34.3.391

[5] Parunak, H. V. D. (1991). Characterizing the manufacturing scheduling problem, Journal of Manufacturing Systems, Vol. 10, No. 3, 241-259, doi:10.1016/0278-6125(91)90037-3

[6] Saravanan, M.; Haq, A. N. (2008). Evaluation of scatter-search approach for scheduling optimization of flexible manufacturing systems, The International Journal of Advanced Manufacturing Technology, Vol. 38, No. 9-10, 978-986, doi:10.1007/s00170-007-1134-5

[7] Luh, P. B.; Feng, W. (2003). From manufacturing scheduling to supply chain coordination: the control of complexity and uncertainty, Journal of Systems Science and Systems Engineering, Vol. 12, No. 3, 279-297, doi:10.1007/s11518-006-0135-5

[8] Teunter, R.; Kaparis, K.; Tang, O. (2008). Multi-product economic lot scheduling problem with separate production lines for manufacturing and remanufacturing, European Journal of Operational Research, Vol. 191, No. 3, 1241-1253, doi:10.1016/j.ejor.2007.08.003

[9] Colorni, A.; Dorigo, M.; Maniezzo, V.; Trubian, M. (1994). Ant system for job-shop scheduling, JORBEL - Belgian Journal of Operations Research, Statistics and Computer Science, Vol. 34, No. 1, 39-53

[10] Kuo, W.-H.; Yang, D.-L. (2006). Minimizing the total completion time in a single-machine scheduling problem with a time-dependent learning effect, European Journal of Operational Research, Vol. 174, No. 2, 1184-1190, doi:10.1016/j.ejor.2005.03.020

[11] Goldberg, D. E. (1989). Genetic Algorithms in Search, Optimization and Machine Learning, Addison-Wesley, Reading

[12] Aloulou, M. A.; Croce, F. D. (2008). Complexity of single machine scheduling problems under scenario-based uncertainty, Operations Research Letters, Vol. 36, No. 3, 338-342, doi:10.1016/ j.orl.2007.11.005

[13] Jabr, R. A. (2005). Robust self-scheduling under price uncertainty using conditional value-at-risk, IEEE Transactions on Power Systems, Vol. 20, No.4, 1852-1858, doi:10.1109/ TPWRS.2005.856952

[14] Chan, F. T. S.; Chung, S. H.; Chan, P. L. Y. (2006). Application of genetic algorithms with dominant genes in a distributed scheduling problem in flexible manufacturing systems, International Journal of Production Research, Vol. 44, No. 3, 523-543, doi:10.1080/ 00207540500319229

[15] Cheng, R.; Gen, M.; Tsujimura, Y. (1996). A tutorial survey of job-shop scheduling problems using genetic algorithms - I. representation, Computers \& Industrial Engineering, Vol. 30, No.4, 983-997, doi:10.1016/0360-8352(96)00047-2

[16] Pranzo, M. (2004). Batch scheduling in a two-machine flow shop with limited buffer and sequence independent setup times and removal times, European Journal of Operational Research, Vol. 153, No. 3, 581-592, doi:10.1016/S0377-2217(03)00264-9 